\title{
CXPRESS: A NOVEL CUSTOMER EXPERIENCE ANALYTICS TOOL
}

\author{
Ioannis Rallis ${ }^{1}$, Ioannis Markoulidakis ${ }^{1}$, George Kopsiaftis ${ }^{1}$, Nikolaos Doulamis ${ }^{1}$, \\ Anastasios Doulamis ${ }^{1}$, Michael Sfakianos $^{2}$ and Kostis Tzanettis ${ }^{2}$ \\ ${ }^{1}$ School of Rural and Surveying Engineering, National Technical University of Athens, Greece \\ 9, Iroon Polytechniou str, Zografou \\ ${ }^{2}$ AppArt S.A., 14, Chalandriou str. 15343, Agia Paraskevi
}

\begin{abstract}
Customer Experience (CxP) is a differentiation strategy for sectors such as telecoms, banks, hotels and retail. Large companies utilize transformation $\mathrm{CxP}$ programs for areas like products, call centers, shops, online, etc. Metrics like the Net Promoter Score (NPS) and "Voice of the Customer" methods are used to collect customer feedback and drive actions. Research conducted in the specific scientific area over the past few years revealed a strong link between NPS and customer loyalty or service revenues. CxPress is a novel customer analytics tool, which was developed based on telecom industry data and advanced data analytics' techniques. Its application could be also expanded in other sectors, e.g. hotels and banks. CxPress tool aims to link the customers' NPS with their satisfaction in key elements, such as product, touchpoint, billing, etc. and their emotional brand engagement. The current paper presents the overall architecture of the CxPress tool, which will support: (a) CxP Strategy design with targets on areas of improvement and differentiation vs. competition, (b) action plan prioritization and verification against strategy targets and (c) fast detection of customer dissatisfaction issues in $\mathrm{CxP}$ operation. A full functional CxPress tool will assist decision makers to adjust their strategic plan to achieve the target NPS.
\end{abstract}

\section{KEYWORDS}

Customer Experience, Net Promoter Score, Machine Learning

\section{INTRODUCTION}

The Customer Experience (CxP) concept was first introduced about three decades ago (e.g. Bolton and Drew, 1991). Since then, it has become a differentiation strategy across industries especially in mature markets with low product differentiation opportunities. Traditional sectors like banks, retail stores, hotels, telecommunications as well as modern digital world corporations (e.g., online stores) recognize the role of $\mathrm{CxP}$ as part of their commercial success. CxP is closely related to customer loyalty management and is measured using survey-based metrics aiming to capture the overall customer satisfaction, purchase or recommendation intention (Bolton and Drew, 1991). As more and more companies are adopting CxP excellence strategies, customers receive higher quality of experience which further raises the bar of CxP competition. In such a competitive environment, $\mathrm{CxP}$ analytics is a topic that attracts the attention of the industry, especially focusing on methods that lead to actionable insights.

$\mathrm{CxP}$ benchmarking studies across industries, indicate the fact that telecommunications are lagging behind other industries, therefore leaving plenty of room for improvement and market differentiation. For that reason, in the recent years, telecom operators increasingly focus and invest on CxP management solutions. However, the $\mathrm{CxP}$ solutions market is still immature allowing the entrance of new players and innovators. In this context, telecom operators are currently focusing on $\mathrm{CxP}$ and big data analytics both for market differentiation and untapped opportunities targeting customer loyalty (reduced churn) and customer value. Forrester's report “Customer Experience Drives Revenue Growth" showed that customer experience leaders achieved compound average revenue growth of $17 \%$ over five years (Manning and Czarnecki, 2016). However, reports suggest that the $\mathrm{CxP}$ investments efficiency is still in the order of $50 \%$ indicating the need 
for methods to better link $\mathrm{CxP}$ metric frameworks with effective $\mathrm{CxP}$ strategies and focused action plans in terms of the results delivered in the areas of customer loyalty and value.

This work presents the overall concept and the main features of the CxPress, a tool which aims to advance the state of the art in Telecommunications CxP analytics, using novel metrics, such as modifications of the widely used Net Promoter Score (NPS) and state-of-the-art machine learning techniques. CxPress based on its innovative solutions aims at the support of essential use cases for telecom operators like: i) customer experience driver's analysis ii) $\mathrm{CxP}$ strategic planning iii) $\mathrm{CxP}$ operational efficiency analysis and iv) linking of CxP KPIs with business KPIs (e.g., churn).

\section{NET PROMOTER SCORE (NPS) INDEX AND RELATED WORK}

Net Promoter Score (NPS) is a well-established methodology (Reichheld, 2003), which is widely adopted across industries as confirmed by CxP professionals (Pointllist, 2019). NPS as an expression of customer's loyalty is based on the so called "NPS question": "How likely are you to recommend [company x] to your friends or colleagues?" The response is provided in the range of 0 (definitely no) to 10 (definitely yes). NPS methodology considers three groups of survey responders depending on the range of their net promoter score (see Table 1). This classification is supported by studies (Reichheld, 2003, Reichheld and Covey, 2006) indicating that Promoters are more loyal and more likely to purchase products and services vs. Detractors.

The NPS KPI for a company is calculated as the difference between the percentage of Promoters and Detractors in the relevant survey:

$$
N P S=\frac{N_{\text {prom }}-N_{\text {detr }}}{N}
$$

Where, $N_{\text {prom }}$ is the number of promoters in the NPS survey, $N_{\text {detr }}$ is the number of detractors in the NPS survey and $\mathrm{N}$ is the total number of NPS survey responders.

Table 1. NPS Customer categorization

\begin{tabular}{ll}
\hline NPS value & NPS Label \\
\hline $9-10$ & Promoter \\
$7-8$ & Neutral \\
$0-6$ & Detractor \\
\hline
\end{tabular}

Apart from the above NPS question, a CxP survey is typically complemented with a set of questions regarding the customer satisfaction on a set of key $\mathrm{CxP}$ attributes such as product experience (service quality, network coverage, tariff plan, billing, etc.), Touchpoint experience (e.g., call center, website, mobile app, shops, etc.), customer lifecycle milestones (e.g., contract renewal), etc. Some surveys include also brand image related attributes (e.g., trust, innovation, etc.) Satisfaction scores also range from 0 to 10 . The resulting satisfaction scores are monitored either based on their mean value or based on the so-called Net Satisfaction Score (NSS) defined in a similar manner to NPS as the difference between the percentage of Attribute Promoters (satisfaction score 9-10) and the percentage of Attribute Detractors (satisfaction score of 0-6).

NPS surveys are performed periodically (with a period ranging from a month up to a year) and they address a random sample of customers in the market. Moreover, NPS surveys are analyzed separately for different market segments (e.g., post-pay, pre-pay, business market segments) because of their different CxP characteristics. In this way, a company may obtain a detailed picture regarding both its own and the market competitors' performance. This analysis is fundamental for a successful CxP excellence program as it provides the necessary customer insights for targeted improvement or market differentiation actions.

A typical analysis of an NPS survey is the so called NPS key drivers' analysis according to which the contribution of the satisfaction scores on the various CxP attributes on the overall NPS is revealed (e.g., Jeske et al. 2011). The analysis is typically based on regression modelling starting from multivariate least squares or logistic regression models. Such a regression analysis apart from the typical challenges (missing survey data handling or data multicollinearities) it can explain a limited amount of the NPS variance (coefficient of determination in the range of 0.4-0.5 for multivariate least squares method). In another research direction, Patrikakis et al. (2007) and Patrikakis and Voulodimos (2008) further extended the driver's analysis, considering several specific factors of the service providers, such as geographical location, customer 
demographics, macroeconomic factors, etc. Finally, a common practice of telecom operators is the exploitation of the Key Performance Indicators (KPIs) framework, which is based on the Quality of Experience (QoE), in order to identify specific service attributes that need to be improved so as to sustain user engagement (Ickin et al., 2019).

Although the NPS analytics tools cover the drivers' analysis part, their methods are bounded by limited accuracy of the applied regression models. Recently, many state-of-the-art tools (Qualtrics, Promoter.io, Medallia) have incorporated Machine Learning (ML) techniques, mainly focusing on sentiment analysis (text analytics) as well as for building prediction models (e.g., for customer churn). However, the application of ML on NPS survey results for deriving customer insights faces serious challenges including the limited available data of the NPS survey and the relatively limited NPS variance of the regression models for several $\mathrm{CxP}$ elements. Moreover, approaches to exploit NPS survey data from different industries so as to better understand the NPS scoring process has not yet proven its value. In this context, CxPress method advances the state-of-the-art since it leads to tangible improvement in the accuracy of the analysis, thus allowing for increased reliability in the CxPress use cases. Such use cases include advanced customer insights (e.g., how Passive Customers are thinking), strategic planning i.e., linking consistentlty the NPS target with respective targets for the CxP elements and allowing for detailed action planning.

In particular, the novel analytics method of CxPress (Rallis et al., 2020): (a) provides a novel classification method according to which customers are classified as "positively", "neutrally" and "negatively" engaged, thus allowing a better understanding of the NPS scoring process and (b) employs state-of-the-art machine learning algorithms leading to increased accuracy of this analysis. This is the core innovation element of CxPress platform that enables the accurate identification of actionable customer insights and provides the essential support to a telecom operator executing a $\mathrm{CxP}$ program with the development of the use cases mentioned in the introduction.

The current paper focuses on the CxPress tool software architecture and development strategy leading to a platform which is expandable in aspects like (a) the user requirements on existing use cases expected to occur during the pilot projects, (b) the introduction of new use cases, (c) the expansion of the tool application to other industries (e.g., banks, hotels, etc.), (d) the ability of the tool to constantly improve its data analytics performance through the incorporation of modern machine learning algorithms and (e) flexibility in integrating and managing company data sources so as to build the target use cases.

\section{CXPRESS DEVELOPMENT METHODOLOGY}

For the implementation of the CxPress software tool, a methodical approach is being followed, in order to minimize the risks and to achieve timely and qualitatively optimized results during the implementation. The methodical approach of the project implementation is based on a step-by-step development of the project's management system and services. In each step the prime goal is to achieve the optimal outcome of the physical object of the project. For the proper management of this project, the PRINCE2 methodology adapted to the needs and data of the project is applied. In addition, Scrum methodology is utilized to develop the individual software systems and algorithms in order to avoid errors and ensure high standard technical implementation. The following table provides the current level of tool development. To upgrade the tool to a full version that can be used to demonstrate the tool's capabilities and commercial utilization, the following sections of work will be required:

Table 2. Step by step analysis of the CxPress tool

\begin{tabular}{ll}
\hline Phases & Description of Work \\
\hline Phase 1: CxPress & $\begin{array}{l}\text { Based on the generalization of the NPS index analysis method, a detailed } \\
\text { specification of the tool's functionality will be developed taking into account } \\
\text { the requirements of the tool user. This specification will focus on use cases }\end{array}$ \\
& $\begin{array}{l}\text { scenarios that provide clear added value to the platform. } \\
\text { Ahase 2: Software }\end{array}$ \\
$\begin{array}{l}\text { A modern Business Intelligence architecture will need to be developed that will } \\
\text { allow easy and flexible data entry from multiple sources (which may vary from } \\
\text { Development }\end{array}$ & $\begin{array}{l}\text { company to company), a convenient user interface (user interface) ) as well as } \\
\text { any ability to support application scenarios suitable for both the new and } \\
\text { inexperienced user and the experienced and demanding user of the tool. The }\end{array}$
\end{tabular}


Phase 3: Operation Control and Pilot Application current version of the tool will be upgraded according to the architecture and specifications of the tool to a full version of the tool which will be suitable for demonstration and pilot application. This version of the tool will be suitable for commercial product promotion with an emphasis on differentiation data compared to other existing methodologies and tools.

The tool that will be developed in the previous work section will go through a thorough check of proper operation based on the application scenarios. Afterwards, there will be demonstration works at various events (e.g. conferences) as well as pilot execution at a productive level, so that its commercial promotion is possible.

\section{CXPRESS OVERALL SYSTEM ARCHITECTURE}

The architecture of the CxPress platform is based on two axes. The first one concerns the possibility of collecting and combining data from multiple sources (heterogeneous data). The second axis concerns the achievement of a level of flexibility and scalability, so that the architecture can incorporate new requirements and usage scenarios. For these reasons, a modern Business Analytics architecture will be adopted, based on the use of Big Data, functionality and easy expansion that will allow the development of new functions at low cost and their rapid entry into the market.

The strategic choice to introduce CxPress as an advanced tool for smart decisions in a company's existing Business Systems (BS) is reflected in the proposed architecture that operates as an open integration framework. In addition, the designed marketing strategy is based on the ability to provide different pricing schemes, tailored per customer and per user scenario, depending on the complexity and the environment in which the tool will be used. Based on the above, the main features of the proposed architecture are presented in the following subsections. As shown in Figure 1, the CxPress platform undertakes to get the input data from all the required sources by various means whether these include downloading files, query on a remote basis or through HTTPs interfaces. The next step is to homogenize process and save the acquired data to the database. The data could be accessed through REST APIs for use by any application, whether it is a web portal, a mobile application, or a third-party application.

CxPress architecture is designed to meet the operating standards of the platform but also to allow a degree of flexibility and scalability if needed due to new requirements and usage scenarios. For this reason, the three-tier model (3-tier) has been selected, which divides the architecture into three levels, completely distinct from each other, giving the platform the necessary flexibility for future extensions.

Specifically, the three levels are the following (Figure 1):

a) Presentation Layer: It is the top level of the three which is the interface of the platform with the end user. It is responsible for the user's interaction with the platform by offering data entry/exit points as well as graphical data representation. The access to the data is granted using an authentication process, while each action requires the proper authorization.

b) Business Logic Layer: It is the intermediate level between the presentation level and the data level. This layer contains all the interface points of the platform, including the external systems that the platform integrates, such as the random number generator and the import / export of files from / to other systems. The presentation level is based on web interface technologies (REST services). Finally, the second layer provides certification/authorization capabilities, through the production and use of random alphanumerics.

c) Data Layer: This layer concerns the communication interface with the database. This level undertakes the creation and execution of queries to the base. This layer is considered separate from the logic level in order to allow for a possible improvements or expansions in the future. mongoDB has been selected as a database, which is a non-relational database that allows the use of dynamic data schemes. This feature could be very useful in applications such as CxPress, which have many different usage scenarios. Furthermore, it allows the reproduction of data by ensuring the required security as well as the extensibility through data sharing. 


\section{DATA IMPORT FUNCTIONALITY}

As mentioned in Section 4, the platform is segregated into three discrete layers, the presentation layer, which consists of the user interface between the user and the platform, the business layer responsible for the overall platform logic and integrations and the data layer for storing and querying the data (Figure 1).

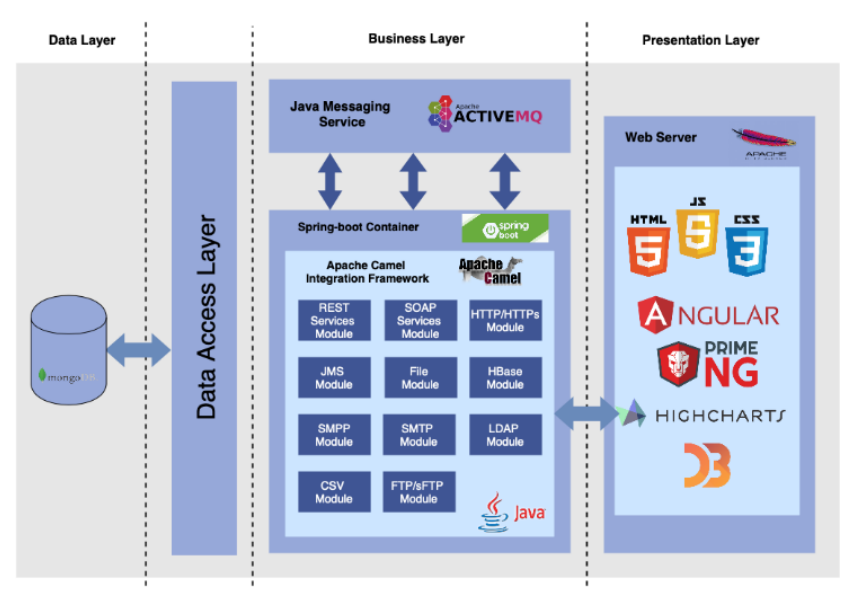

Figure 1. Adopted technologies for the proposed CxPress framework

CxPress platform integrates a variety of different data sources, which will feed the platform with the information defined by the proposed user scenarios. CxP data sources may include all IT and Service Platform systems of a company including Customer Relation Management (CRM), Billing, Website, Mobile App. Value Added Services Platforms, Campaign management systems, Sales management systems, network management systems, etc. For that reason, CxPress should support flexible and fast-track data integration either for the platform setup activities or for integrating additional data sources on-the-fly so as to gradually enhance the platform functionality in existing customers.

The collection of input data is supported by the corresponding functionality (Data Ingress), which consists of a set of web services, transfer and processing of files, questions in remote databases and more, depending on the customer's systems. The Data Import module is followed by the Data Staging function, in which the data is properly formatted and stored so that it is ready to be processed. This architecture allows the integration of users with local environment of different specifications and requirements, which affect (exclusively) the input and preparation of data. Data Import functionality supports the following features:

Data Input Function: This function is responsible for collecting data from different sources with possibly different shapes (data-schemas) and automation processes. In order for this stage to provide maximum functionality, the tool supports the following data input capabilities: (i) Files uploaded via FTP / sFTP or through the use of web services by the client's organization, (ii) excel files, uploaded via FTP / sFTP or through the use of web services by the customer's organization. The limitation of 65536 lines for Microsoft Excel is taken into account in this case, (iii) Tissue Services (REST / SOAP), (iii) Interfaces for OSS / FMS systems.

Data Preparation Mode: All data is converted to an intermediate format. The conversion process is designed to be flexible based on the selected user scenarios and to allow for the implementation of parallel processes for the sufficient handling of big-data sources.

A web-based application that can be accessed via mobile devices (responsive) will be implemented within the CxPress framework. Through this application the presentation level will interact with the logic level using web services. The ability to manage the level of presentation separately allows for the implementation of different applications for mobile devices, without modifying the logic layer. All connections are made through a secure transport layer, which ensures the overall security of the application. Regarding CxPress technologies, depending on the level (data, logic and presentation), different open-source technologies will be used (see Figure 1). 
The CxPress platform is able to support several profiles and user roles (management, IT, marketing, customer care etc.) of different level of expertise. To that end, the CxPress tool interface is user friendly and simple to use without compromising any of the data mining functionalities and platform capabilities which concern the experienced users. Finally, the CxPress tool provides a number of data processing and presentation and the ability to extract tables (dashboards) and reports with the required data.

\section{CXPRESS USER INTERFACE}

The CxPress tool provides a user-friendly interface, which consist of the following main pillars:

- $\quad$ The Login page (Figure 2)

- The Data Entry page (Figure 2)

- Data Analysis module (Figure 3).
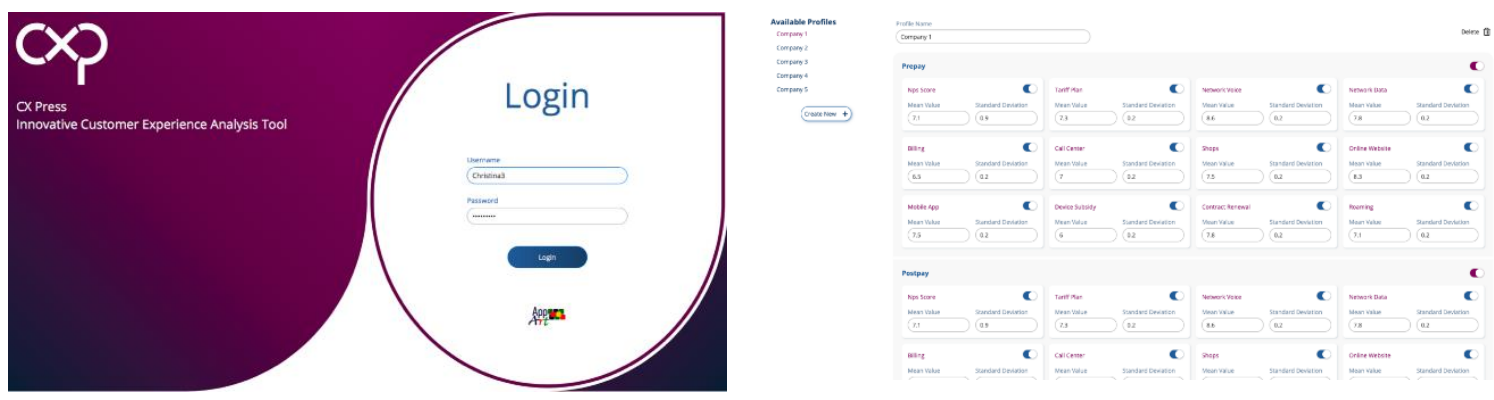

Figure 2. Login and Data entry pages

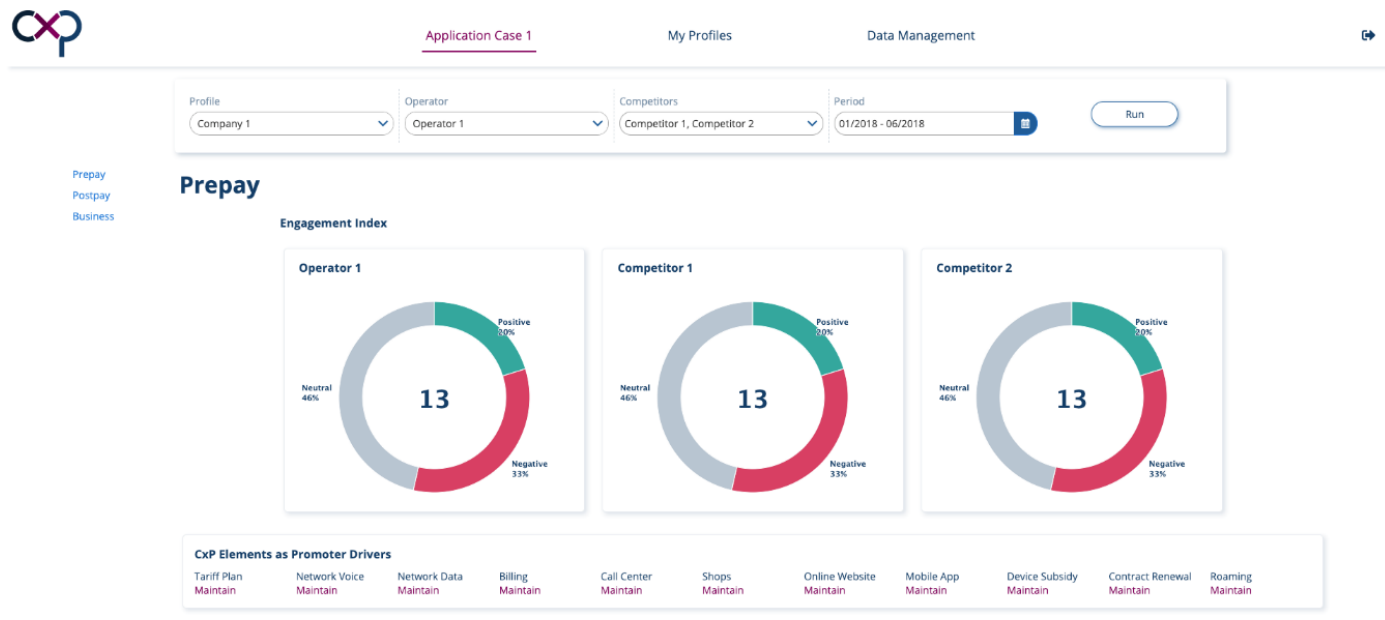

Figure 3. Indicataive data analysis screenshot from the CxPress tool

\section{BUSINESS LOGIC LAYER \& THE MACHINE LEARNING MODULE}

The business logic layer has full access to the company data delivered through the data layer so as to apply specific use cases. The key enabler of this module is the Data Analytics library of the platform where both state-of-the-art and the novel CxPress data analytics techniques are made available. Such techniques allow for descriptive, prescriptive and predictive analytics. The core element of the Business Logic Layer is the Use Case Management module where the logic of each individual Use Case of the tool is being implemented and delivered to the user of the CxPress tool through the UI layer (e.g., dashboard presentation, reporting, etc.) 
The Use Cases of the Platform refer to the following key areas:

(a) NPS survey analysis and customer insights (NPS forecasting, Passive Customers insights)

(b) NPS Strategic Planning (Consistent NPS and CxP Elements Targeting Framework)

(c) Valuation and Prioritization of CxP Action Plan

(d) CxP Operations Analysis and Delivery of CxP Targets

(e) Linking of $\mathrm{CxP}$ with Business Performance (e.g., customer churn, customer value, etc.)

State-of-the-art churn propensity models rely on regression modelling and lately on machine learning algorithms applied on a wide range of customer data (e.g., demographic, product usage, revenue, transactions data including complaints, customer lifecycle, etc.) to predict the churn probability. Due to the complexity of the problem and the dynamic market environment the accuracy of these models may vary significantly. As operators invest in initiatives related to prevention of churn by targeting the high churn risk customers the accuracy of these models has a direct financial impact.

CxPress implements the above use cases based on state-of-the-art data analytic and machine learning techniques (Figure 4). The CxPress approach exploits all available data sources and builds use case specific models which continuously improve either due to the additional data feeds or by the enhancement of the data analytics techniques. The innovation of CxPress is that depending on the information available for each customer the model is flexible enough to provide predictions for the customer behavior based on partial information elements (e.g., NPS survey response, satisfaction survey right after a transaction, online transactions data, customer reaction to commercial campaigns, customer purchase, usage patterns, etc.).
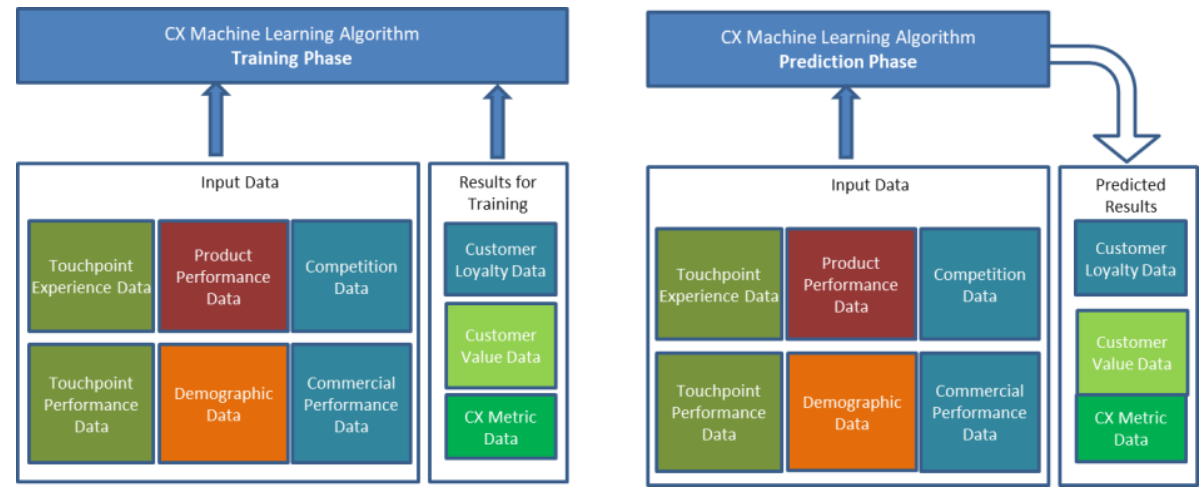

Figure 4. CxPress analytics framework encompassing machine learning algorithms

CxPress platform is open to innovation and extension of the developed method to the following directions:

(a) Application of the survey analytics method to other metrics: The method has already been successfully applied to customer metrics other than NPS like Customer Satisfaction Score (CSAT), Brand Image survey as well as Employee Satisfaction Survey.

(b) Application of the method to industry sectors beyond telecoms: the novel method of CxPress is currently in piloting phase for a company in the Banking sector while the project is going to further extend the method so as to cover the hospitality sector. Other sectors can probably be addressed in the future (e.g., Power Supply companies, Healthcare Sector, etc.)

\section{CONCLUSION \& FUTURE WORK}

During the CxPress project the machine learning algorithms will be further enhanced with data available from the pilots so as to ensure that the algorithm becomes generic enough to cover different operators activated in different markets. To accelerate this process, recent developments in Generative Adversarial Networks (GANs) will be exploited. GANs provide the ability to generate data with the characteristics of an existing data set allowing for faster training of a machine learning algorithm. In terms of the algorithm itself various options will be examined (e.g., as Support Vector Machines, Long Short Term Memory, Convolutional Neural Networks, Deep Belief Networks, Restricted Boltzmann Machines) so as to select the most appropriate one for the problem considered. 


\section{ACKNOWLEDGEMENT}

This research has been co-financed by the European Union and Greek national funds through the Operational Program Competitiveness, Entrepreneurship and Innovation, under the call RESEARCH - CREATE INNOVATE (project code: T1EDK-05063).

\section{REFERENCES}

Aksoy, L., Buoye, A., Aksoy, P., Larivière, B., \& Keiningham, T. L., 2013. A cross-national investigation of the satisfaction and loyalty linkage for mobile telecommunications services across eight countries. Journal of Interactive Marketing, 27(1), 74-82.

Bolton, R. N., \& Drew, J. H., 1991. A multistage model of customers' assessments of service quality and value. Journal of consumer research, 17(4), 375-384.

Gentile, C., Spiller, N., \& Noci, G., 2007. How to sustain the customer experience: An overview of experience components that co-create value with the customer. European management journal, 25(5), 395-410.

Ickin, S., Ahmed, J., Johnsson, A. and Gustafsson, J., 2019, June. On Network Performance Indicators for Network Promoter Score Estimation. In 2019 Eleventh International Conference on Quality of Multimedia Experience (QoMEX) (pp. 1-3). IEEE.

Jeske, D.R., Callanan, T.P. and Gui, L., 2011. Identification of key drivers of Net Promoter Score using a statistical classification model. Efficient decision support systems: practice and challenges from current to future. Shanghai: InTech, pp.145-62.

Manning, H. and Czarnecki, D., 2016. Customer Experience Drives Revenue Growth, 2016. Business case: The Customer experience ecosystem Playbook. Forrester Research Inc, p.7.

Medallia, Text Analytics, https://www.medallia.com/platform/text-analytics/

Patrikakis, C., Karamolegkos, P., Voulodimos, A., Abd Wahab, M.H., Taujuddin, N.S.A.M., Hanif, C., Pareschi, L., Riboni, D., Weber, S.G., Heinemann, A. and Cheung, S.C.S., 2007. Security and privacy in pervasive computing. IEEE Pervasive Computing, 6(4), pp.73-75.

Promoter.io, Analyze NPS with Machine Learning, https://www.promoter.io/blog/analyze-nps-with-machine-learning-ai/

Qualtrics Net Promoter Score Software, https://www.qualtrics.com/uk/customer-experience/nps-software/

Rageh Ismail, A., Melewar, T. C., Lim, L., \& Woodside, A., 2011. Customer experiences with brands: Literature review and research directions. The Marketing Review, 11(3), 205-225.

Rallis, I., Markoulidakis, I., Georgoulas, I., Kopsiaftis, G., 2020. A novel classification method for customer experience survey analysis. Proceedings of the 13th ACM International Conference on PErvasive Technologies Related to Assistive Environments, Corfu, Greece.

Reichheld, F.F., 2003. The one number you need to grow. Harvard business review, 81(12), pp.46-55.

Reichheld, F., 2006. The microeconomics of customer relationships. MIT Sloan management review, 47(2), p.73.

Reichheld, F.F. and Covey, S.R., 2006. The ultimate question: Driving good profits and true growth (Vol. 211). Boston, MA: Harvard Business School Press.

Voulodimos, A.S. and Patrikakis, C.Z., 2008, August. Using personalized mashups for mobile location based services. In 2008 International Wireless Communications and Mobile Computing Conference (pp. 321-325). IEEE. 